\title{
Norois
}

Environnement, aménagement, société

$234 \mid 2015$

Dynamique des milieux humides, éducation à l'environnement, pratiques festives, tourisme et patrimonialisation

\section{La stabilité limnique dans le bassin de la Vienne}

La place des plans d'eau au sein des milieux et des sociétés depuis le XIX ${ }^{\mathrm{e}}$ siècle

Pascal Bartout et Laurent Touchart

\section{OpenEdition}

\section{Journals}

Édition électronique

URL : https://journals.openedition.org/norois/5581

DOI : $10.4000 /$ norois. 5581

ISBN : 978-2-7535-4218-1

ISSN : $1760-8546$

Éditeur

Presses universitaires de Rennes

Édition imprimée

Date de publication : 30 juin 2015

Pagination : 65-82

ISBN : 978-2-7535-4183-2

ISSN : 0029-182X

Référence électronique

Pascal Bartout et Laurent Touchart, «La stabilité limnique dans le bassin de la Vienne », Norois [En

ligne], 234 | 2015, mis en ligne le 30 juin 2017, consulté le 13 janvier 2022. URL : http://

journals.openedition.org/norois/5581; DOI : https://doi.org/10.4000/norois.5581

(c) Tous droits réservés 


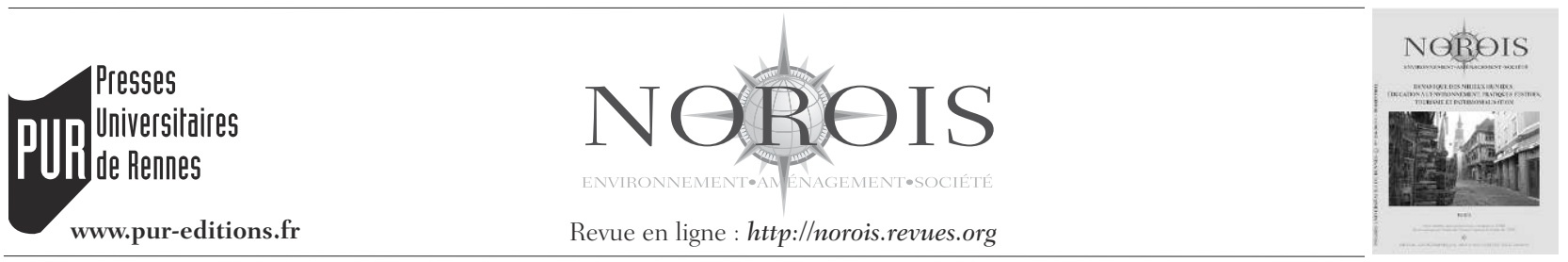

\title{
La stabilité limnique dans le bassin de la Vienne La place des plans d'eau au sein des milieux et des sociétés depuis le $\mathrm{XIX}^{\mathrm{e}}$ siècle
}

\author{
Limnic Stability in Vienne's Basin (France) \\ The Place of Waterbodies in Environments and Societies since the $19^{\text {th }}$ Century
}

\author{
Pascal Bartout* ${ }^{*}$, Laurent Touchart
}

\author{
* Auteur correspondant \\ EA 1210 CEDETE, ITP HCS, Collegium UFR LLSH, Université d'Orléans, 10 rue de Tours - 45065 OrLÉANS \\ cedex, France (pascal.bartout@univ-orleans.fr) (laurent.touchart@univ-orleans.fr)
}

Résumé : La connaissance des plans d'eau français souffre de trois biais scientifiques rendant inopérants ou peu efficaces certains de leurs modes de gestion actuels : méconnaissance des chiffres et des localisations exactes par les organismes chargés de leur administration, traitement comme un hydrosystème fluvial et non comme un limnosystème, omnipotence de la démarche biologique chez les représentants du pouvoir, au détriment des hommes, alors que les étangs et les autres petits plans d'eau artificiels sont des objets créés par et pour l'homme. Les auteurs présentent ici une approche permettant de comprendre sans a priori la place des plans d'eau dans des paysages de manière diachronique (1830 et 2005), afin d'envisager les territoires lentiques selon leur degré de stabilité limnique. À travers l'étude du bassin de la Vienne, les auteurs font apparaître des multitudes de réponses différenciées selon les mutations sociologiques et économiques observées, sur un fond de qualité agronomique liée à la nature du sous-sol. La partie aval est restée globalement stable, alors que l'amont cristallin et son contact avec le bassin sédimentaire ont connu des modifications importantes.

\begin{abstract}
The knowledge of French waterbodies is affected by three scientific bias making some current management systems ineffective: (i) the ignorance of their number and exact locations by the State or decentralized administrative bodies, (ii) the interpretation as fluvial hydrosystems and not as limnosystems; and (iii) the omnipotence of the biological approach among officials and representatives of power to the detriment of the social approach, although ponds and other little artificial waterbodies are objects created by and for humans. Here, the authors present a diachronic approach (1830 and 2005) allowing the understanding without a priori of the place of waterbodies in landscape to consider lentic territories according to their level of limnic stability. Through the case study of Vienne's basin, the authors reveal the multitude of differentiated answers according to sociological and economical changes, and study the presence or absence of waterbodies according to agronomical features. The basin downstream remains rather stable, whereas the crystalline part upstream and its contact with the sedimentary basin have been largely modified.
\end{abstract}

Mots clés : Plans d'eau - Étangs - Bassin de la Vienne - Étude diachronique - Paysage

Keywords: Waterbodies - Ponds - Vienne's basin - Diachronic study - Landscape 


\section{INTRODUCTION}

La Directive-cadre européenne sur l'eau (DCE2000) et sa transcription dans le droit français à travers la loi sur l'eau et les milieux aquatiques (LEMA) de 2006 ont pour ambition d'améliorer l'état écologique des eaux européennes et françaises et elles imposent de réussir à le faire d'ici 2015. Les eaux de surface à surveiller sont formées à la fois de cours d'eau et de plans d'eau. Pour ce qui est de ces derniers, les autorités françaises, par l'intermédiaire des agences de l'eau, en reconnaissent 456 (statut de «masse d'eau plan d'eau »), ne considérant que ceux qui sont d'origine naturelle ou bien, si ce n'est le cas, ceux de grande taille. Cela revient à ne pratiquement reconnaître que les lacs naturels, avant tout concentrés sur les marges montagneuses du pays, et les barrages-réservoirs de plus de 50 ha, en général récents (en grande majorité postérieurs à la Seconde Guerre Mondiale), les moins intégrés dans le paysage et la culture ancestrale des habitants.

Or nous avons montré que les plans d'eau français de plus d'un are dépassaient le nombre de 554000 (Bartout et Touchart, 2013), O. Scher (2008) estimant pour sa part à environ un million le nombre de collections d'eau stagnante de plus de $10 \mathrm{~m}^{2}$. Ce vertigineux décalage, en partie voulu, en partie subi, entre la réalité et la prise en compte officielle ne risque-t-il pas de mener à des incohérences de gestion, si ce n'est d'obtenir l'inverse de l'effet escompté et de laisser se dégrader finalement une partie des masses d'eau?

Pour tenter de répondre à cette question majeure, nous partons de l'idée que les liens entre la société française et les petits plans d'eau artificiels sont pluriséculaires et ancrés culturellement, offrant à chaque territoire une variété de réponses aux contraintes géographiques. Pour étayer cette hypothèse, nous avons fait le choix d'une étude diachronique entre deux moments clés de l'histoire limnique récente, à savoir la première partie du XIX siècle et la situation actuelle, à l'échelle d'un espace de plus de $20000 \mathrm{~km}^{2}$ représentatif de la diversité géographique et limnique française, le bassin de la Vienne. Les résultats permettront d'identifier les territoires ayant connu une continuité dans la place occupée par les petits plans d'eau, que nous appelons stabilité limnique, marquée par une acceptation locale forte, les distinguant de ceux où les modifica- tions opérées au cours des dernières décennies ont fait naître un nouveau modèle paysager qui rompt l'équilibre ancien et nécessite un temps d'adaptation du milieu et des hommes.

\section{LE RECUL TEMPOREL LIMNIQUE POUR UNE GESTION CONTEMPORAINE APPROPRIÉE}

À l'instar de L. Lespez (2012) en BasseNormandie et M. Franchomme (2008) dans le Nord de la France, nos travaux s'inscrivent dans une dynamique de compréhension des milieux humides et de leurs dynamiques propres, non restituables par transfert de modèles. Pour ce faire, il est nécessaire de s'intéresser au rôle joué par les sociétés du passé sur leur environnement et ainsi comprendre les dynamiques paysagères affectant les territoires étudiés. Parmi les patrimoines hydrauliques et hydrologiques, cette production scientifique se focalise sur les plans d'eau et notamment les étangs.

En effet, plus que tout autre objet limnique, l'étang est, parmi les plans d'eau artificiels, la masse d'eau la moins concernée par la seule entrée écologique correspondant aux directives et réglementations actuelles (Dufour et Piégay, 2009, Finger-Stich, 2013). Ce point est autant la résultante de l'absence de terme équivalent en anglais, langue des directives européennes, qu'une volonté franco-française. Ainsi, pour O. Cizel (2013), « les textes peuvent, à l'occasion, se référer spécifiquement aux mares, mais ils ne font pas de distinction précise entre les étangs et les lacs, qui sont assujettis bien souvent à un régime identique ». Leurs recensements, cartographies et compréhensions sociales ne sont donc pas des priorités dans la politique de l'eau nationale.

Ceci nous conduit à rechercher les causes de l'écart existant entre l'attachement d'une part importante de la population aux étangs et leur dévalorisation presque systématique, donc non proportionnée, dans les écrits administratifs et techniques.

\section{L'étang, le grand incompris limnique français}

Nous regrouperons les raisons supposées de cette discordance en un triptyque provocateur, constitué d'une réalité d'évidence, d'une ignorance et d'un dogme. 
Une tautologie, car les plans d'eau français, presque tous anthropiques, ont par essence même éloigné depuis plus ou moins longtemps les bassins fluviaux de leur état de nature originelle, en augmentant la part des écosystèmes lentiques aux dépens des milieux lotiques. Mais les limiter à cette évidence ne permet que de s'offusquer et réclamer leur suppression. Dépasser ce truisme autoriserait pourtant à chercher des solutions intermédiaires, concertées et proposées avec discernement. Puisque ces étangs existent de fait par centaines de milliers sur notre territoire, ne vaudrait-il pas mieux les aménager en assumant leur caractère lentique que les nier?

Une méconnaissance, car assimiler le plan d'eau à une portion mise à mal de cours d'eau revient à ignorer son fonctionnement propre, donc les moyens les plus appropriés de l'accompagner vers l'amélioration. Naturel ou artificiel, parfois plus ancien dans le second cas que dans le premier, grand ou petit, le plan d'eau est un limnosystème, un écosystème lentique dans lequel la composante verticale du cycle trophique prend le pas sur les contrastes d'amont en aval (Angelier, 2000). En plan d'eau, l'étagement des processus devient la référence de fonctionnement, même dans les plus petits étangs, donc celle de l'aménagement raisonné, par exemple dans les choix de système d'évacuation d'eau dépendant de la profondeur de prélèvement (Touchart, 2007). Faut-il feindre de l'ignorer, ne considérer le plan d'eau que comme un accident exclusivement perturbateur d'une normalité fluviale à atteindre à tout prix, consolider la puissance corporatiste de certaines organisations professionnelles? Ne peuton pas, comme tentent de l'évoquer les géographes limnologues, "considérer le plan d'eau comme un possible moteur du réseau hydrographique et de l'économie régionale » (Touchart et al., 2014)?

Une vérité établie, car ladite " continuité écologique » (article 6 de la LEMA, traitant des « obligations relatives aux ouvrages »), au lieu de rester un fait concret se matérialisant de multiples manières sur des terrains différents, est devenue une théorie englobante que plus personne n'a le droit de mettre en doute, ne serait-ce que pour mettre en lumière certains cas particuliers ou, mieux, faire entendre le fait que la restauration de la «continuité écologique » n'est pas forcément synonyme de marche vers le «bon état écologique ». En effet, ce dernier dépend des caractéristiques stables de chaque milieu identifié (cours d'eau, prairie humide, plan d'eau, etc.), alors que la «continuité écologique» exclut toute rupture topographique au sein du talweg. De fait, il nous semblerait sensé d'admettre que sa restauration partout, qui forme un plan national depuis 2009 et est pratiquement devenu la raison d'être de l'ONEMA, incite à la destruction de petits ouvrages hydrauliques tels que les biefs, les moulins, les canaux, les étangs, présents depuis des siècles, intégrés dans le paysage et pour lesquels le profil du cours d'eau s'est réajusté depuis longtemps. D'ailleurs, le nouveau référentiel national des obstacles à l'écoulement (RNOE) n'inventorie pas les ruptures naturelles, dont certaines entravent pourtant le transit sédimentaire et les migrations des organismes vivants. Quant à la stratégie d'effacement, elle ne vise aucunement les grands barrages, car la Realpolitik concède, sinon leur utilité économique, du moins la puissance des groupes de pression qui les soutiennent.

On ne peut comprendre la mise en place de ce triptyque sans une connaissance approfondie de l'évolution dans le temps de la répartition géographique des petits plans d'eau artificiels. Estimer leur date de création, le contexte social, économique et paysager de l'époque, permet d'éviter de nier leur existence ou les considérer comme de simples accidents de l'histoire faciles à effacer. Saisir qu'un marais naturel pouvait précéder l'étang artificiel permet de comprendre que le cours d'eaux vives n'est pas toujours l'état originel de la nature. Accepter le fait qu'un étang a pu être mis en eau par des moines du haut Moyen Âge relativise l'échelle de temps de la dynamique fluviale, ses actions et rétroactions. Le très récent lac de Vallon, né de façon naturelle au milieu du $\mathrm{xx}^{\mathrm{e}}$ siècle d'un glissement de terrain des montagnes du Chablais barrant une vallée, est-il obligatoirement bienfaisant pour le transit sédimentaire fluvial puisque l'homme n'y est pour rien et a-t-il incontestablement droit au statut de « masse d'eau plan d'eau » pour être bien géré, alors qu'on refuse cette possibilité à un étang millénaire du Limousin?

Il est donc impératif de comprendre la genèse de ces étangs, mais également de toutes les autres formes de plans d'eau, afin de les percevoir tels qu'ils sont et non tels que l'on voudrait qu'ils fussent. Pour cela, la suite de cette étude se focalisera sur la compréhension de l'évolution numérique des 
plans d'eau, point déjà analysé (Bartout, 2010) en Limousin grâce au croisement de multiples archives (locales, régionales et nationales), de cartographies de différentes époques et d'observations paysagères sur le terrain. Deux dates, 1830 et 2005, ont semblé pertinentes, car elles correspondent peu ou prou aux deux derniers maxima numériques observés pour les plans d'eau (figure 1) et deux outils cartographiques précis permettent d'en dresser le portrait, à savoir le cadastre napoléonien et le référentiel de Bartout.

Cependant, compte tenu des limites du premier, cette approche cartographique se fera uniquement par commune et non par bassin-versant. De même, si notre démarche concerne les plans d'eau dans leur ensemble, nous n'avons de recul scientifique qu'au sujet des étangs, car eux seuls avaient une valeur marchande et ont donc été identifiés textuellement ou cartographiquement. Aussi cette étude se focalisera avant tout sur ces objets et n'abordera la question des autres plans d'eau que lorsqu'il s'agira d'expliquer les composantes paysagères régionales.

\section{L'apport scientifique d'une approche diachronique stagnustre}

Explicitées dans deux articles récents (Bartout, 2011, Bartout et Touchart, 2013), les méthodologies ayant conduit à la constitution des deux inventaires sont différentes et mériteront donc d'être discutées afin de comprendre ce qui est transposable et ce qui ne l'est pas.

\section{Le référentiel des plans d'eau de Bartout}

Le référentiel des plans d'eau de Bartout n'est pas un simple inventaire car il s'agit d'un Système d'Information Géographique renseigné sur un certain nombre de critères, issu lui-même d'un traitement de la couche «SURFACE_EAU » de la BD Topo de l'IGN. Il s'agit donc d'un traitement photogrammétrique de clichés aériens pris entre 2001 et 2005 pour l'ensemble de la France métropolitaine, complété par un travail de terrain ayant permis de renseigner plus de 15000 plans d'eau (en l'occurrence dans les bassins de l'Indre, du Cher, de la Charente, de la Dordogne et de la Vienne). Nous considérons donc que les résultats issus de ces traitements, même si ces derniers ont été effectués et publiés postérieurement, correspondent à un état des lieux de la France en 2005.

Les vérifications effectuées ont permis de mettre en évidence un indice de corrélation de 0,986 pour le recensement et la localisation des plans d'eau de plus d'un are (surface minimale en deçà de laquelle
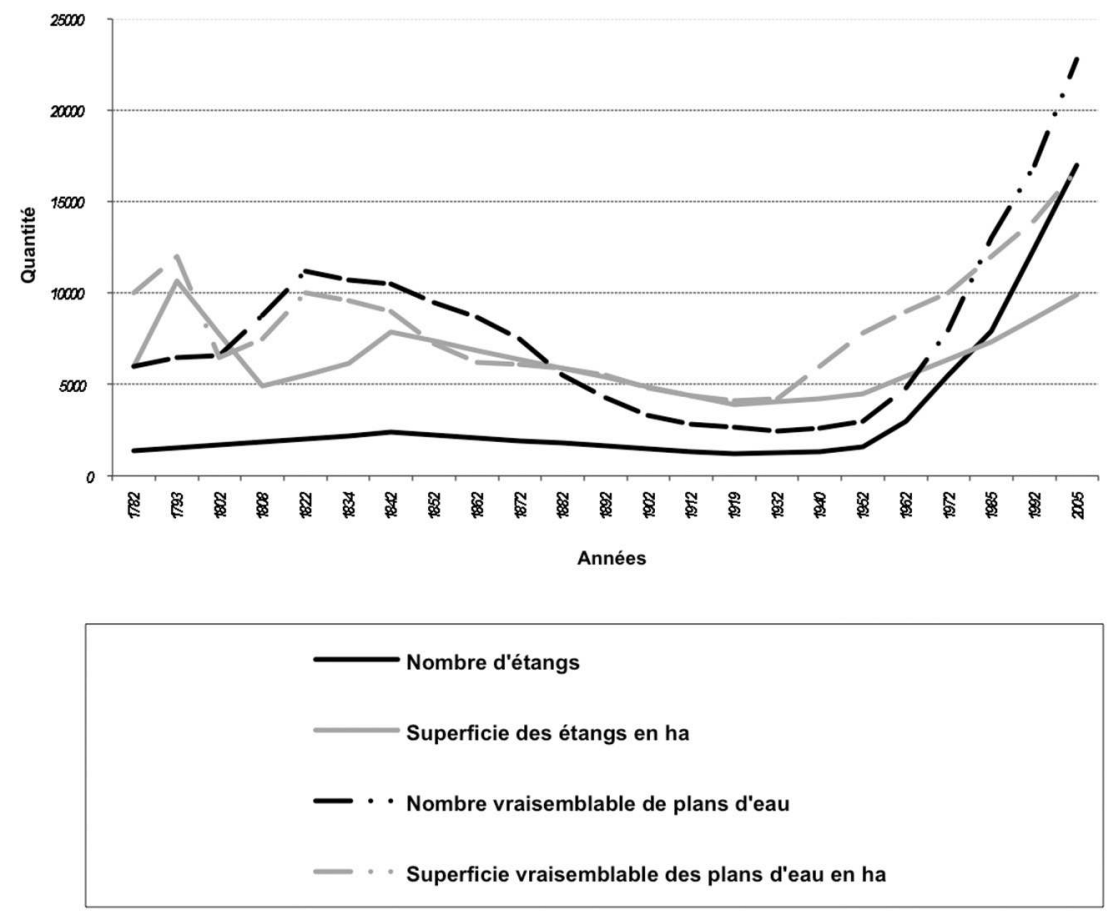

Figure 1 : Estimation des variations en nombre et en superficie totale (en ha) des plans d'eau au cours des 2 derniers siècles en Limousin (d'après Bartout, 2010)

Estimation of the variation of waterbodies number and their total surface area during the two last centuries in Limousin 
les incertitudes pesant sur la réalité du fait lentique sont trop fortes).

\section{L'inventaire des plans d'eau issu des relevés du cadastre napoléonien}

Le Cadastre Napoléonien, aussi nommé Ancien Cadastre, est un outil foncier élaboré sur le terrain entre 1807 et 1850 pour les territoires appartenant à l'espace français métropolitain de l'époque. Construit après l'instauration des règles cartographiques de la Commission topographique de 1802, sa «langue topographique » est transposable à celle actuelle, permettant des comparaisons numériques et surfaciques avec des produits cartographiques postérieurs malgré un temps de saisie et de traitement extrêmement long puisque chaque plan d'eau doit être vectorisé après calage de l'image raster du cadastre le concernant.

La fiabilité de cet outil est également très importante (mais difficilement quantifiable dans le détail) car les plans parcellaires du cadastre adoptent une échelle cartographique fine, allant du $1 / 500^{\mathrm{e}}$ au $1 / 2000^{\mathrm{e}}$, et que l'objet foncier que constitue l'étang est une source d'impôts à percevoir, donc celui-ci est cartographié. Néanmoins, la fiabilité des relevés est plus faible pour les mares, non soumises aux taxes, car les géomètres topographes n'avaient pas pour fonction de décrire le paysage. Il existe donc vraisemblablement une sous-estimation des plus petits plans d'eau.

\section{Les limites méthodologiques de cette comparaison temporelle}

Une transposition exacte des résultats des deux inventaires est impossible pour deux raisons

La première provient de l'étalement temporel de la constitution du cadastre napoléonien, à savoir plus de 40 ans. Or, comme le graphique 1 a pu le montrer, cette période coïncide avec des fluctuations numériques importantes. Il était donc opportun d'harmoniser les résultats issus du comptage des plans d'eau afin de ne pas fausser l'analyse cartographique en surestimant les relevés correspondant aux années 1825-1840 et en sous-estimant ceux des années 1807-1820. Pour cela, nous avons pu mettre en place un mécanisme visant à harmoniser les données grâce à la présence de deux cadastres sur certaines communes. Élaborée à partir de 13 doubles cadastres du département de l'Indre, cette méthode a été confortée a posteriori par 62 doubles cadastres du département de la Vienne correspondant à des milieux géographiques variés. Ainsi, en voulant faire un état des lieux lentique en 1830, nous avons multiplié les relevés faits par:

-2 pour les recensements de 1808 à 1810 ;

$-1,8$ pour ceux de 1811 à 1815 ;

- 1,6 pour ceux de 1816 à 1820 ;

- 1,4 pour ceux de 1821 à 1825 ;

- 1,2 pour ceux de 1826 à 1830 ;

- 1 pour les relevés postérieurs à 1830 et ceux qui étaient sans date.

La deuxième limite ne concerne pas le décompte des plans d'eau mais l'aspect sémantique : un étang de 2005 correspond-il à un étang de 1830 et viceversa? Cette réflexion vaut également pour les mares.

Pour les travaux de 2005, ne connaissant pas la profondeur de tous les plans d'eau français alors même que la détermination de celle-ci est le critère d'entrée principal quant au découpage sémantique et fonctionnel de ce que sont une " mare », un « étang » et un « lac » (Touchart, 2007, Bartout, 2010), nous avons opté pour le découpage classique au niveau international, en fonction de la superficie des plans d'eau, avec deux césures à 0,1 ha et à 100 ha. Est ainsi «mare» tout plan d'eau de superficie inférieure à 0,1 ha, « lac » tout plan d'eau de superficie égale ou supérieure à 100 ha, et « étang » un plan d'eau compris entre les deux seuils.

Cependant, bien qu'il soit possible d'obtenir la superficie des plans d'eau sur le cadastre napoléonien, le traitement à l'échelle d'un territoire de $20000 \mathrm{~km}^{2}$, rend la chose irréalisable. Aussi, la séparation sémantique entre " mares » et «étangs » (car il n'y a pas de « lacs») s'est fondée sur les critères suivants : est «étang » tout plan d'eau identifié comme tel sur les plans cadastraux et ce quelle que soit sa superficie, mais également tout plan d'eau de superficie supérieure à 10 ares ou muni d'un système de vidange clairement mentionné. Tout autre plan d'eau est rangé dans la catégorie «mare ».

La conséquence immédiate de ces limites méthodologiques est la non-adéquation complète entre ce que nous entendrons par «mare » et «étang » dans les résultats à venir. Il ne s'agira donc pas de s'attarder sur le chiffre exact de l'étude mais plus sur la tendance qu'elle procure. 


\section{L'ÉTUDE DES ÉTANGS DU BASSIN DE LA VIENNE, UN CAS D'ÉCOLE}

\section{Le choix du bassin de la Vienne}

Les régions administratives ne possédant pas de compétences «plans d'eau » même si la valorisation de ceux-ci peut être de leur fait, nous avons opté pour une approche scalaire par bassin-versant. Celle-ci possède l'avantage d'une continuité hydrologique et d'une logique de gestion par l'intermédiaire des Agences de l'Eau. Parmi les territoires des six agences de bassin de métropole, un domine l'ensemble par ses caractéristiques lentiques : en effet, il possède actuellement près d'un étang sur deux (48,74\% exactement), pour $30 \%$ de la superficie hexagonale, soit 122474 étangs sur les 251289 mis en évidence par Bartout (2015a) en France. Il s'agit du bassin Loire-Bretagne (figure 2).

À l'intérieur de cet ensemble, nous avons tenté de sélectionner le bassin-versant régional le plus représentatif des continuités et discontinuités lentiques mises en évidence depuis quelques années (à savoir la pente, la nature du substrat et des formations superficielles, le type d'écoulement de surface et le rang de Strahler pour la partie physique, et la proximité d'une ville ou de voies de communication rapides, la valorisation économique et les mutations paysagères pour la partie humaine). À cet égard, trois possibilités existaient et permettaient d'envisager la régionalisation de phénomènes spatiaux et temporels : les bassins du Cher, du Maine et de la Vienne. Parce qu'il cumule le plus d'avantages scientifiques permettant d'appréhender au mieux la place des plans d'eau au sein des milieux hier et aujourd'hui en France continentale, le choix du bassin de la Vienne (figure 3), avec ses 43000 objets lentiques, dont 19000 étangs, s'est imposé.

Tout d'abord, il se situe géographiquement dans deux des trois régions administratives mises en avant dans la classification des plans d'eau de Bar-

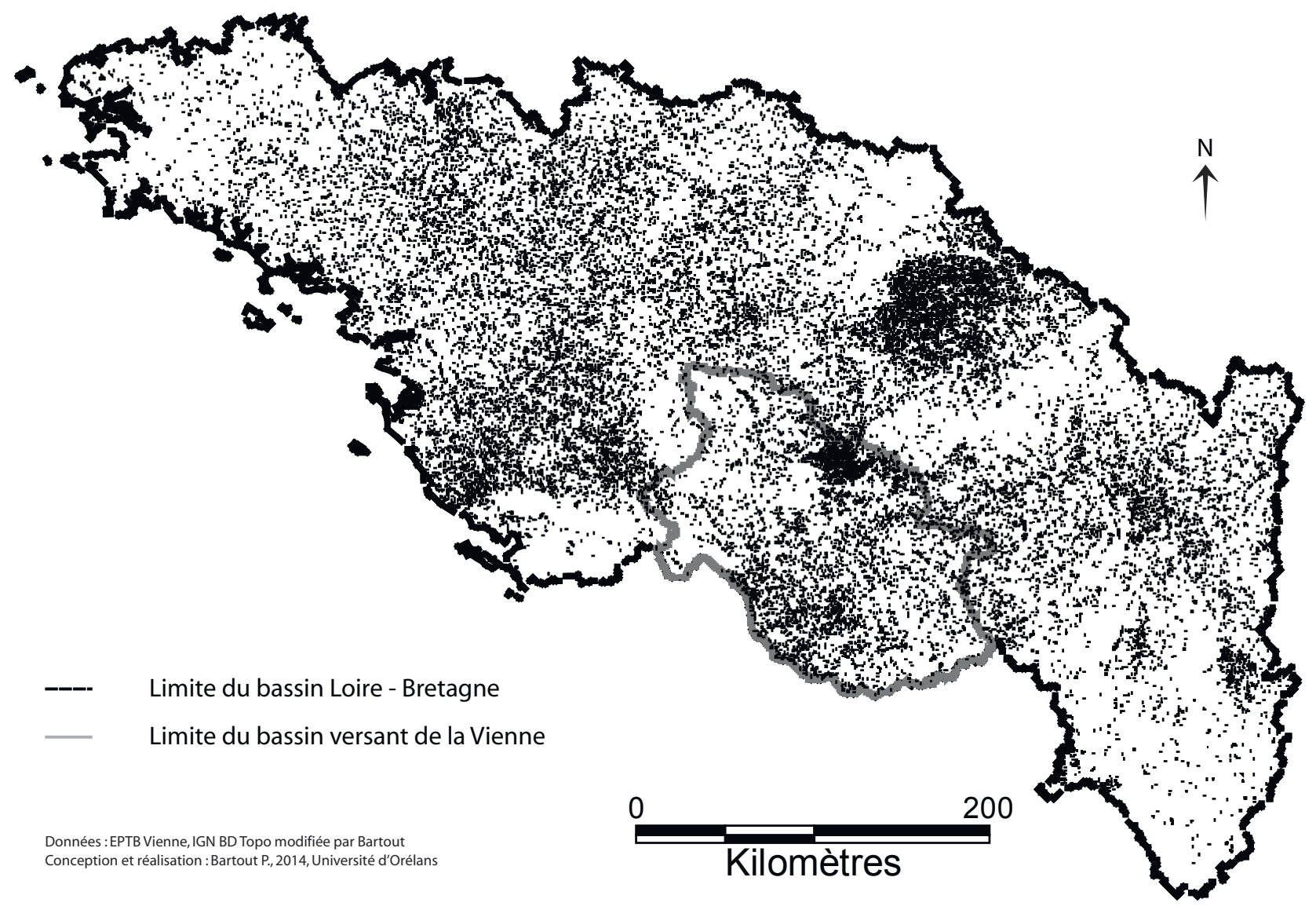

Figure 2 : Les étangs du bassin Loire - Bretagne en 2005 Loire - Bretagne basins ponds in 2005 
Figure 3 : Carte de localisation du bassin de la Vienne Vienne's basin

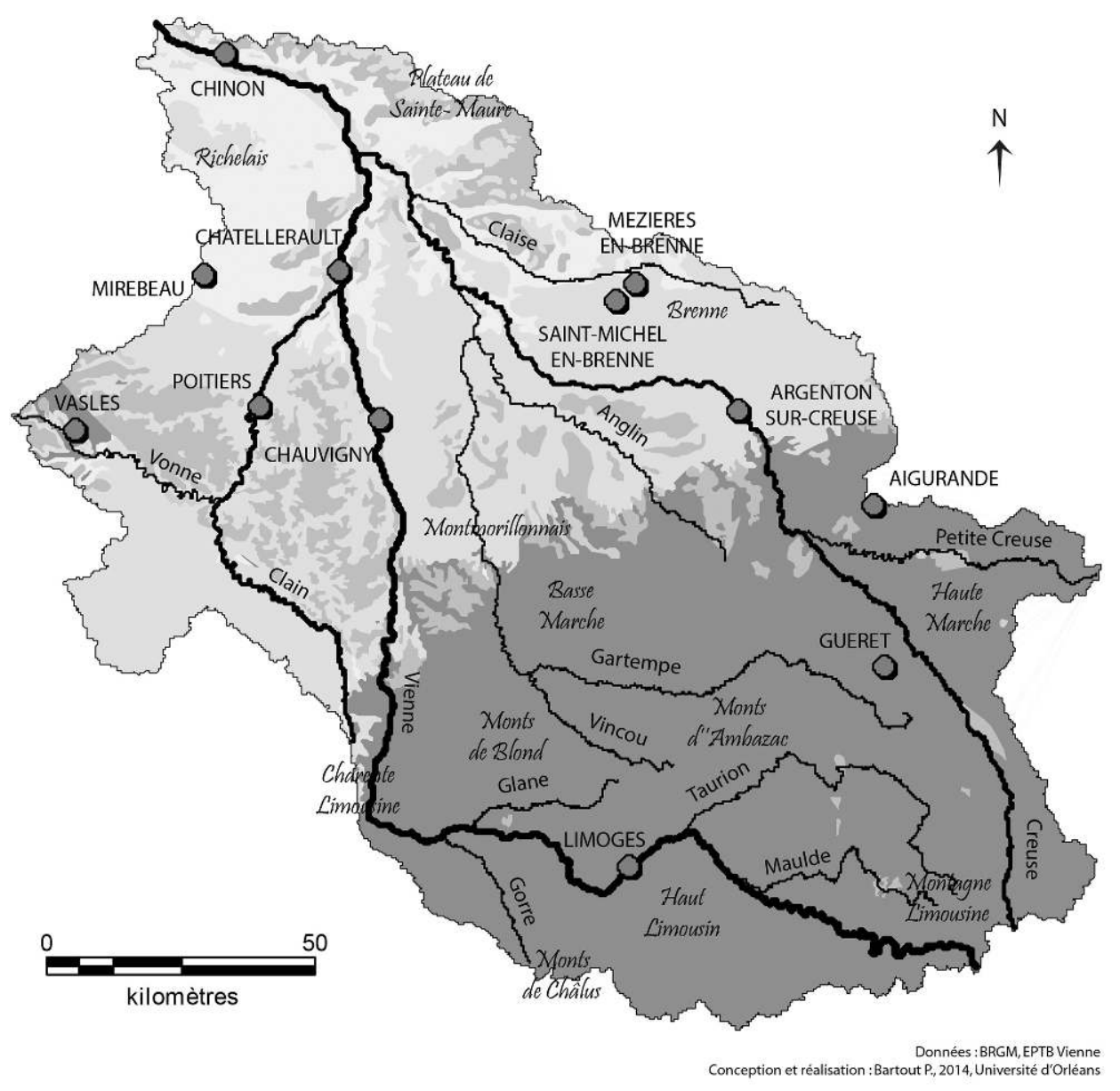

Géologie

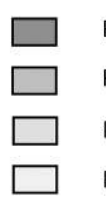

Roches métamorphiques, plutoniques et volcaniques Roches sédimentaires à dominante argileuse dont argiles à silex Roches sédimentaires à présence argileuse

Roches sédimentaires dénuées d'argiles

Hydrographie

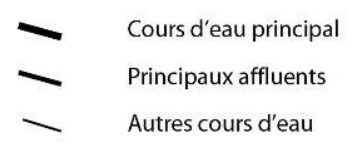

$\begin{array}{cl}\text { Richclais } & \text { Régions géographiques évoquées } \\ & \text { Villes évoquées }\end{array}$

la Loire, offrant la possibilité d'accueillir de multiples écosystèmes lotiques et lentiques différents et des liens variés entre hommes et eaux. À ce facteur topographique s'ajoute en outre la présence de roches et de dépôts superficiels aux caractéristiques très marquées, à l'origine d'une inégale densité de plans d'eau (Bartout, 2015c). Enfin, le bassin de la Vienne a connu de multiples études géographiques centrées sur ses plans d'eau au cours des dernières décennies (Aldomany et al., 2013, Ardillier-Carras, 2007, Azaroual et al., 2012, Balabanian et Bouet, 
1989, Bartout, 2010, 2012, Bédoucha, 2011, Benarrous, 2009, Bernard, 2008, Brunaud, 2007, Busnel, 1985, Carlini, 2006, Maleval, 2002, Papon, 2007, Touchart, 2002, Touchart et Graffouillère, 2004), voire antérieurement (Perpillou, 1940, Vacher, 1908), permettant aujourd'hui de mieux comprendre les contextes physiques et socio-économiques de cet ensemble hydrologique.

\section{Les étangs du bassin de la Vienne depuis le haut Moyen Âge}

Ces nombreuses études permettent de retracer assez fidèlement l'histoire des étangs au sein du bassin de la Vienne. Cette approche se révèle indispensable car elle permet de saisir les raisons qui nous ont conduits à produire une étude de géographie limnologique diachronique.

Même si les premiers étangs du bassin de la Vienne remontent vraisemblablement au $\mathrm{IV}^{\mathrm{e}}$ siècle, les premières véritables concentrations d'étangs sont à mettre à l'actif des ordres monastiques. En Brenne, les moines de Saint-Cyran et de Méobecq (La Véronne, 1967) ont été, dès le VII ${ }^{\mathrm{e}}$ siècle, les précurseurs d'une activité de drainage puis de mise en valeur des terres marécageuses par la double fonction permise par l'étang, à savoir l'agriculture (assec) et la pêche (évolage). Dans la partie limousine, les moines de Grandmont dans les monts d'Ambazac (Barrière, 1998) ont donné naissance aux premières concentrations stagnustres de la vallée du Vincou autour de l'étang de Tricherie.

À ces moines se sont adjoints les seigneurs qui ont très tôt compris l'intérêt financier que pouvait leur procurer un étang. Les concentrations en étangs dépendaient donc avant tout dans un premier temps du maillage d'abbayes et de châteaux d'un territoire. En cela, le Poitou fut plus marqué par l'aspect ecclésiastique avec une activité pêche développée en lien avec le respect des jours de jeûne, alors que le Limousin fut plus influencé par les seigneurs laïcs très nombreux sur cette zone frontière au Moyen Âge, qui virent dans l'étang un moyen d'allonger le fonctionnement saisonnier du moulin bannier dans les parties amont des bassins-versants par le système de l'éclusée.

Cette répartition dura jusqu'au XvIII ${ }^{\mathrm{e}}$ siècle, même si certaines bourgeoisies urbaines s'immiscèrent dans le marché des étangs (Cassan, 2006). Le maxi- mum d'étangs fut vraisemblablement atteint à la fin du XVII ${ }^{\mathrm{e}}$ siècle, moment où les pratiques religieuses commenceront à décliner.

Entre la fin du XVIII ${ }^{\text {e }}$ siècle et les années 1960, les parties amont et aval du bassin de la Vienne connurent des destins lentiques différents jalonnés de péripéties communes. Ainsi, si le décret du 14 frimaire an II (1793), qui visait à assécher les étangs, car détenus par les ordres privilégiés, toucha l'ensemble du bassin de la Vienne, la réponse des sociétés locales fut différente. En Limousin, les sociétés paysannes remirent en état les étangs et en créèrent de nouveaux pour répondre aux besoins d'autarcie agricole ainsi qu'aux besoins industriels de la France par l'intermédiaire des moulins sur étangs. En Poitou, bien que quelques établissements industriels virent le jour comme la forge de Corbançon en Brenne, le nombre d'étangs ne cessa de chuter sous l'effet d'une mode hygiéniste (assainir les terres marécageuses sources de miasmes) puis agraire qui voulait faire de la Brenne le grenier à blé du Berry (Vacher, 1908). Avec un décalage de 30 à 50 ans, le Limousin des étangs fut touché par ce phénomène en lien avec l'exode rural et la généralisation des énergies fossiles qui avaient supplanté la force motrice générée par l'eau.

La carte des densités d'étangs en 1830 (figure 4) procure un état des lieux stagnustre à la croisée de ces dynamiques numériques positives et négatives. Elle fait apparaître une césure territoriale d'axe sudouest - nord-est où le Poitou et les hauts-plateaux limousins apparaissent peu dotés en étangs (même s'ils dépassent bien souvent la moyenne nationale de l'époque ${ }^{1}$ ), contrairement à l'espace intermédiaire surdoté d'où émerge l'espace brennous.

Si la Brenne s'est de nouveau couverte d'étangs dès les années 1930, elle le doit avant tout à sa fonction piscicole. Aujourd'hui, ces grands étangs constituent la trame brennouse reconnue internationalement (site Ramsar) à l'intérieur de laquelle quelques étangs d'agrément contemporains sont venus se glisser.

Tel n'est pas le cas de la partie limousine qui a connu un réveil tardif mais spectaculaire car, comme l'a montré la figure 1, le nombre de créations depuis les années 1960 est vertigineux (à l'échelle du Limousin, ce sont en moyenne 350 créations

1. 0,03 étang par $\mathrm{km}^{2}$ après traitement des travaux de Rougier de la Bergerie (1819). 
Figure 4 : La densité d'étangs par $\mathrm{km}^{2}$ par commune du bassin de la Vienne en 1830

Ponds density by $\mathrm{km}^{2}$ by commune in Vienne's basin in 1830

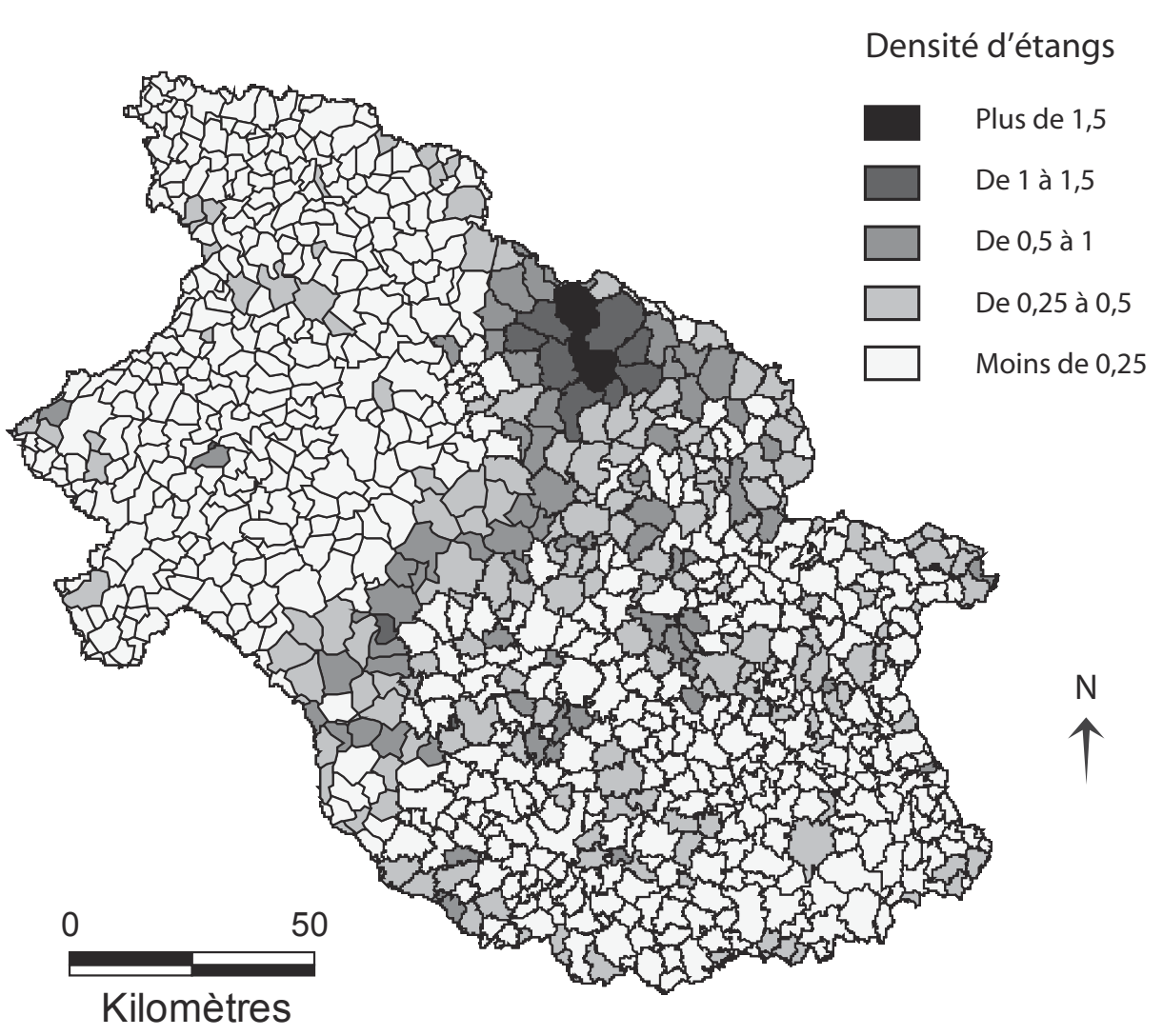

Données : Archives départementales, IGN BD Geofla, EPTB Vienne Conception et réalisation : Bartout P., 2014, Université d'Orléans d'étangs par an pendant 40 ans!). L'agrément est le moteur de cette (r)évolution stagnustre et aujourd'hui les campagnes limousines proches (en temps) de l'agglomération limougeaude sont couvertes de petits plans d'eau à proximité de résidences secondaires ou principales.

\section{État des lieux des étangs du bassin de la Vienne en 2005}

Ainsi, en 2005, avec une densité de 0,9 étang par $\mathrm{km}^{2}$, le bassin de la Vienne possède une concentration d'étangs qui est le double de la densité française $(0,46)$. Cette caractéristique ne concerne cependant pas tous les recoins du bassin de la Vienne et la figure 5 nous permet de comprendre que le fait stagnustre concerne avant tout le bassin de la Creuse et de la Vienne amont et secondairement le bassin du Clain. Dans le détail (figure 6), nous voyons nettement ressortir deux espaces aux fortes densités, à savoir la Brenne au nord-est du bassin et un territoire à cheval sur la Charente limousine et les monts d'Ambazac et de Blond au sud-ouest.
Si l'on cherche à mieux comprendre la place réelle prise par les étangs dans l'espace et les sociétés, il est utile de compléter cette approche numérique par une approche surfacique. Avec plus de 21000 hectares en eau, le bassin de la Vienne contribue pour plus de $8 \%$ de la superficie en eau nationale (alors qu'il représente moins de $4 \%$ de la superficie hexagonale), soit une stagnucité (= pourcentage de la superficie d'un territoire en eau d'étang) de $1 \%$ (contre 0,48 nationalement). Ces fortes superficies en eau se retrouvent géographiquement dans les mêmes espaces que pour la densité, à la nuance près que le bassin de la Creuse ressort davantage (figure 7) et que les très fortes superficies se trouvent en Brenne et dans des vallées comme le Vincou ou la Glane (figure 8). Ces caractéristiques démontrent que les étangs sont multiples à l'intérieur du bassin de la Vienne (étendus en Brenne, peu étendus en Haut-Limousin par exemple) et ces morphologies particulières sont adaptées à des besoins économiques différents (pisciculture en Brenne, loisirs autour de Limoges), qui plus est évolutifs au cours des siècles en fonction des besoins humains. 


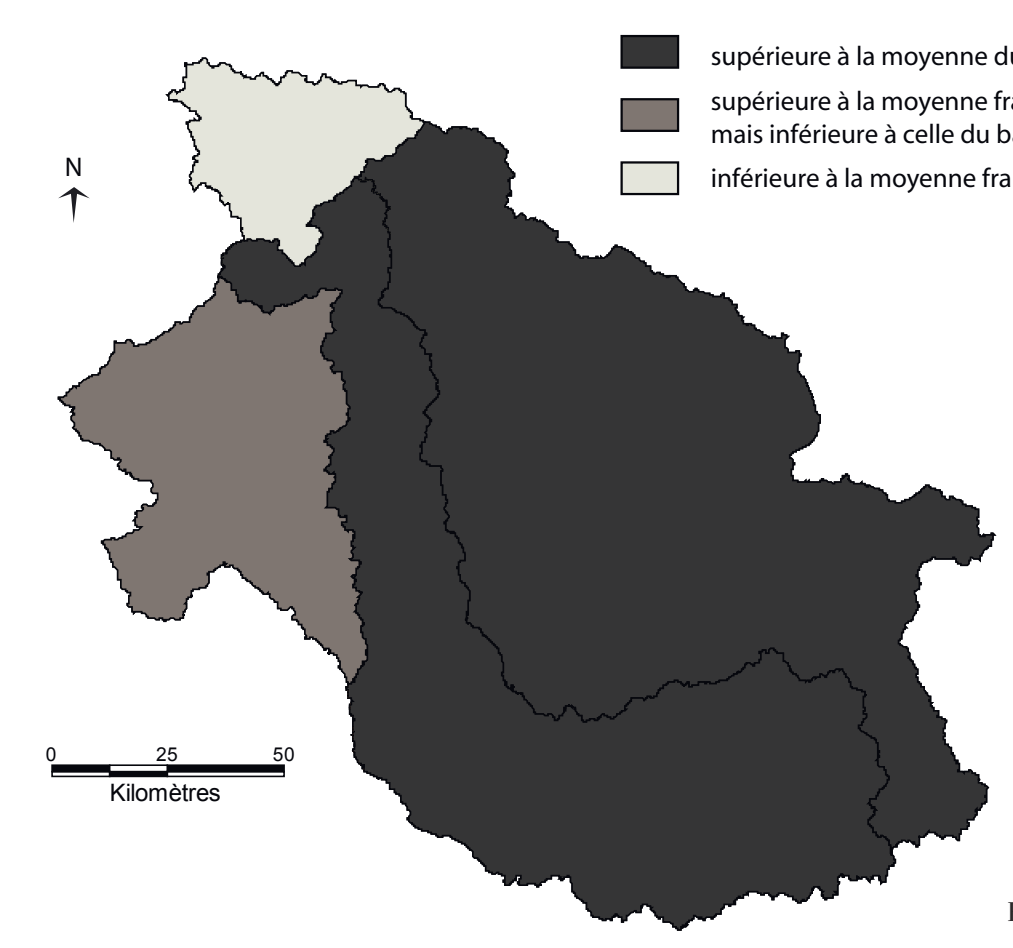

ise de données : Bartout, BD Topo mod., EPTB Vienne Inception et réalisation : Bartout P., 2014

Figure 5 : La densité de plans d'eau par grand bassin Waterbodies density by big basin

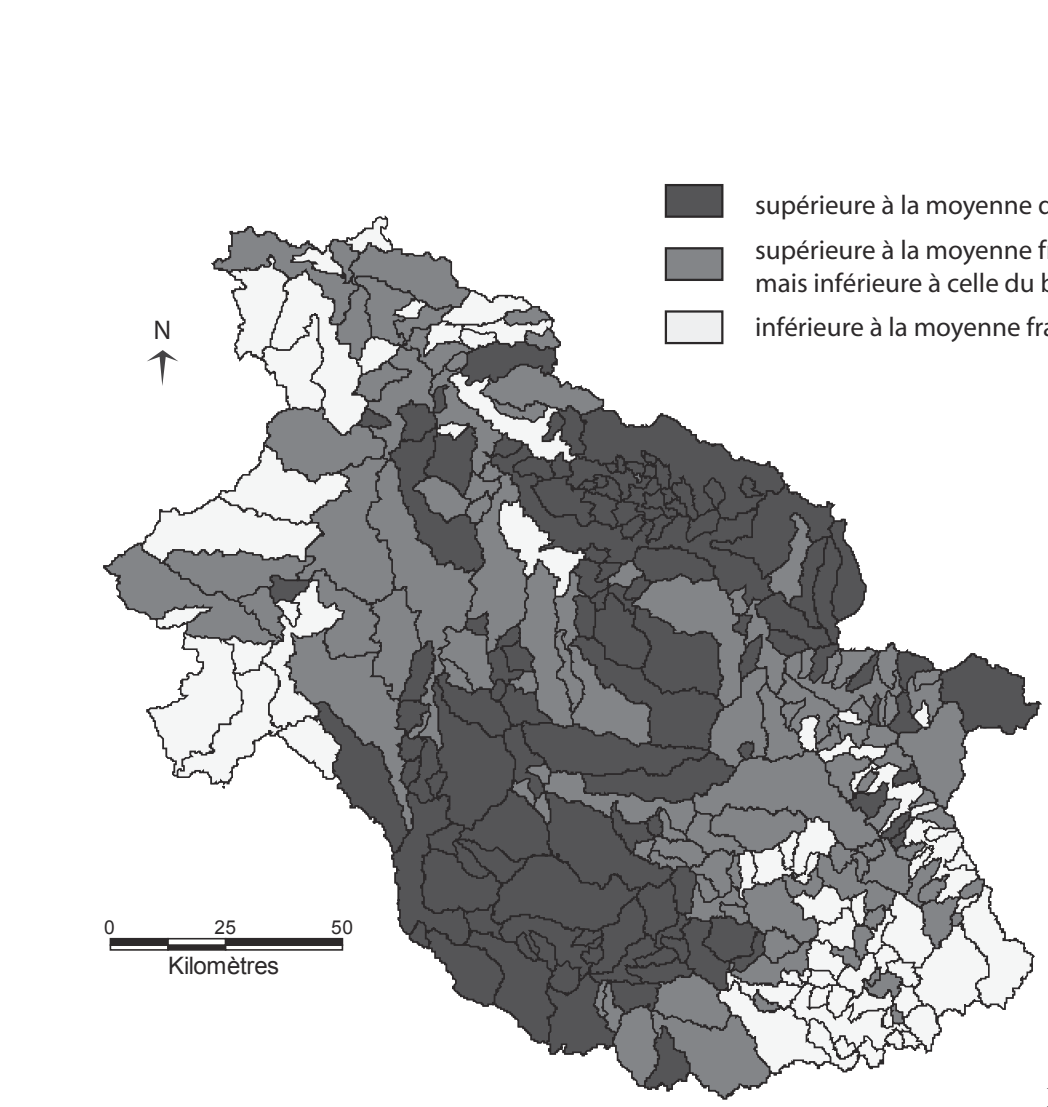

Base de données : Bartout, BD Topo mod., EPTB Vienne

Figure 6 : La densité de plans d'eau par sous bassin Conception et réalisation: Bartout P., 2014

Waterbodies density by small basin 


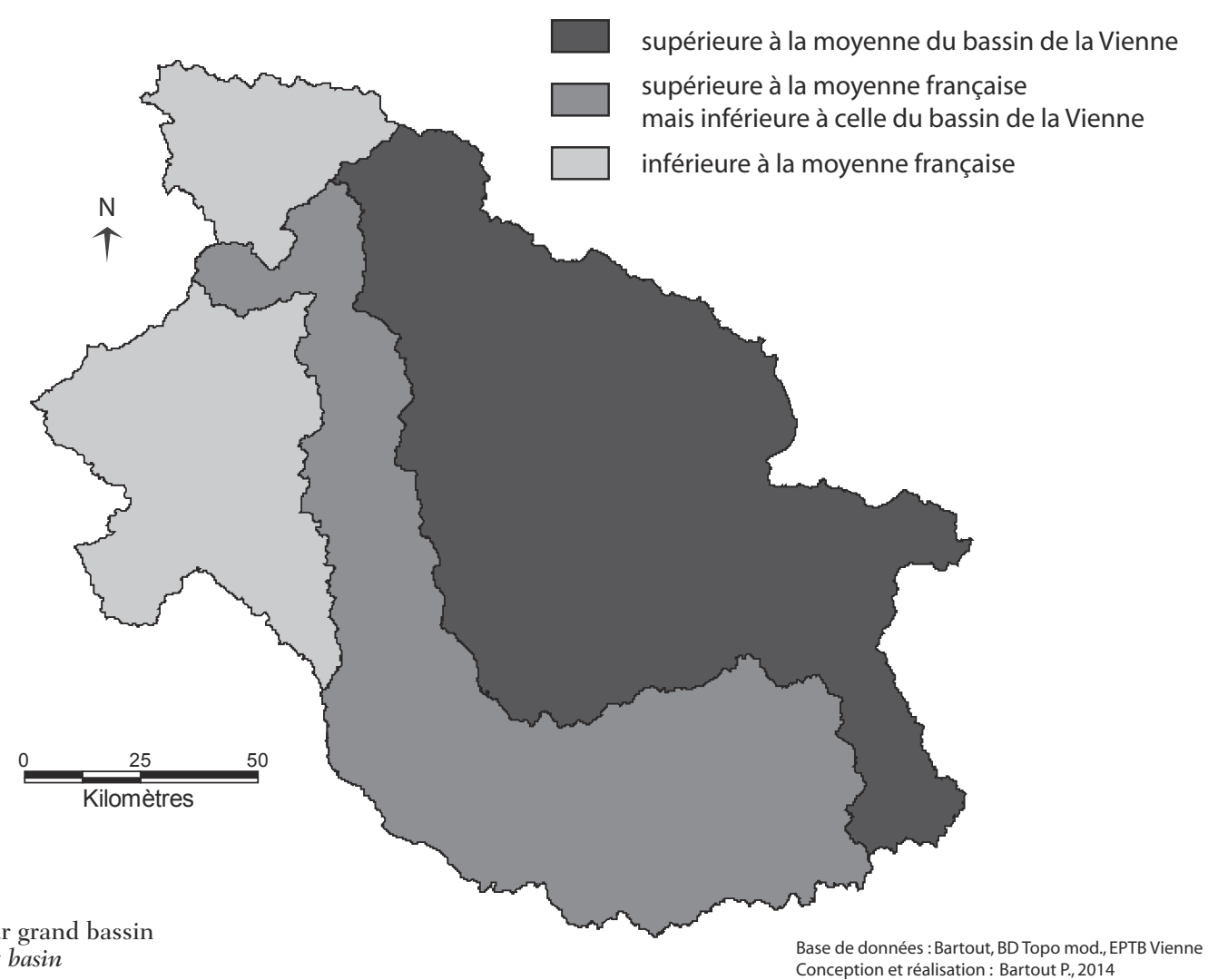

Figure 7 : La stagnucité par grand bassin Stagnucity by big basin Base de données : Bartout, BD Topo mod., EPTB Vienne

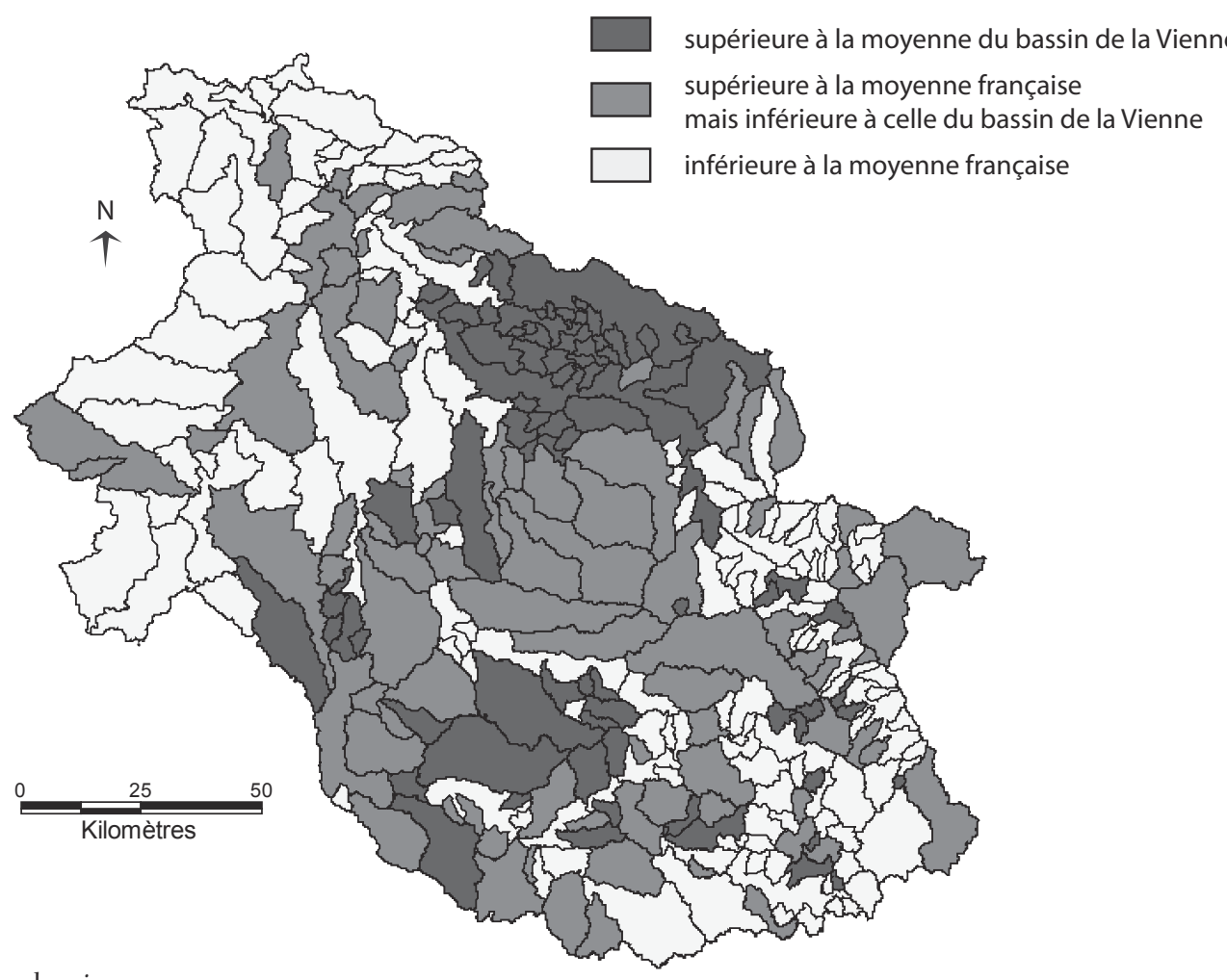

Figure 8 : La stagnucité par sous bassin

Base de données : Bartout, BD Topo mod., EPTB Vienne Stagnucity by small basin

Base de données : Bartout, BD Topo mod., EPTB
Conception et réalisation : Bartout P., 2014 


\section{La densité de plans d'eau, indicateur numérique de stabilité paysagère}

Le bassin de la Vienne est passé de 29150 plans d'eau en 1830 à 42863 en 2005, soit une augmentation de $47 \%$. Cette augmentation numérique est avant tout le fait des étangs, passés de $20 \%$ du total lentique en 1830 à $45 \%$ en 2005, les mares ayant quant à elles connu une stagnation numérique, passant de 23594 en 1830 à 23934 en 2005.

La figure 9 ci-après prenant en compte les deux types de plans d'eau permet de mieux cerner les dynamiques lentiques des territoires du bassin de la Vienne.
Celle-ci met en avant la césure d'axe sud-ouest - nord-est séparant le bassin de la Vienne en trois grands secteurs géographiques, précédemment mise en évidence pour les étangs (figure 4), où l'espace intermédiaire constitue toujours le lieu préférentiel d'installation des plans d'eau, mais celle-ci est complétée par une extension méridienne au nord et au sud venant perturber cette logique ancienne.

Ainsi, au sud, c'est la très forte hausse de la densité des bas-plateaux limousins à l'ouest de Limoges qui vient rompre le schéma ancien en se couvrant beaucoup plus rapidement de plans d'eau que la Montagne limousine à l'Est. Au nord, en revanche,

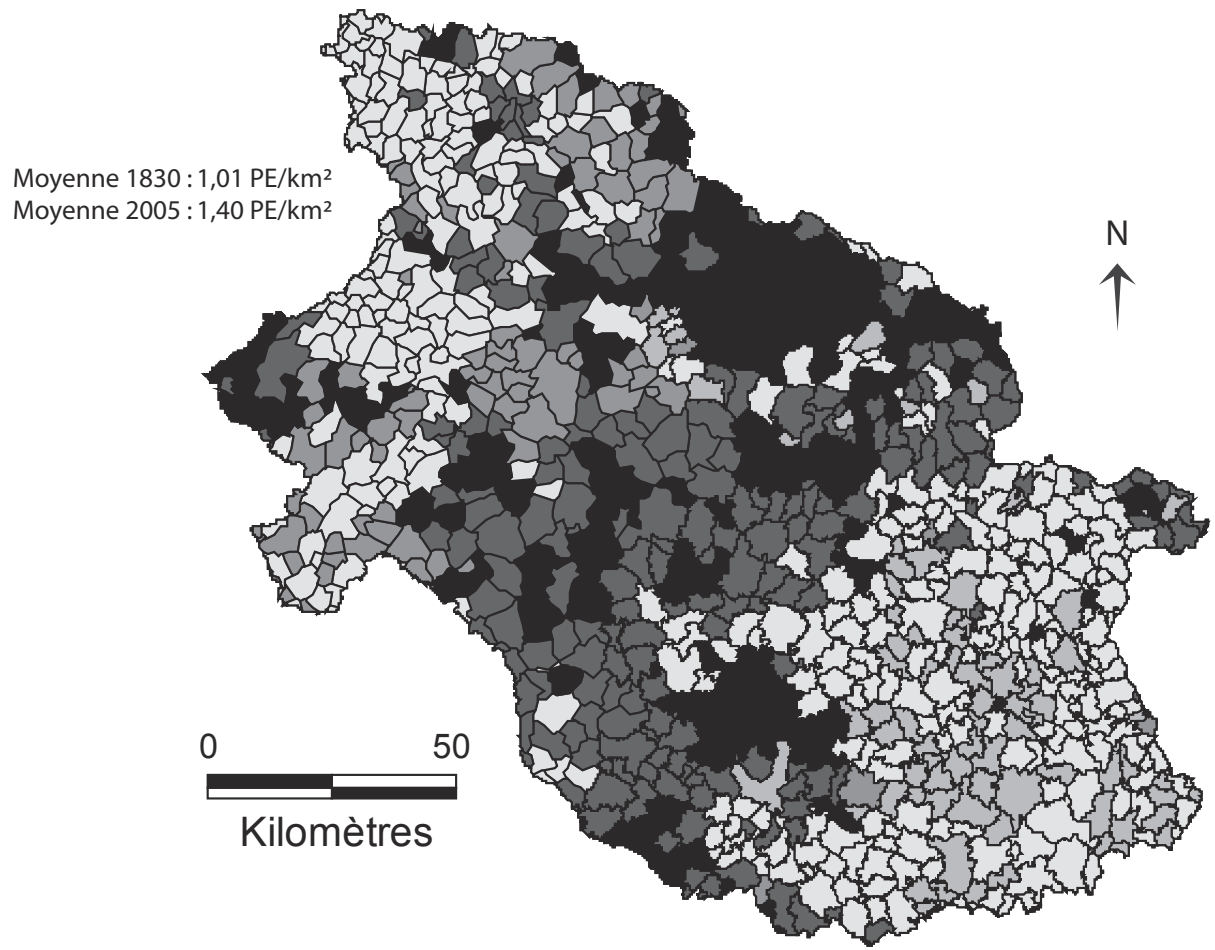

Dynamiques des densités de plans d'eau entre 1830 et 2005

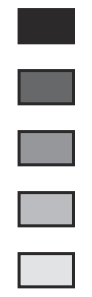

Densité toujours au-dessus de la moyenne

Forte hausse de la densité permettant de passer au-dessus de la moyenne en 2005

Stagnation de la densité entre 1830 et 2005 au niveau de la moyenne

Forte baisse de la densité entraînant un passage en dessous de la moyenne en 2005

Densité toujours en-dessous de la moyenne

Données : Archives départementales, IGN Bd Topo modifiée, IGN BD Geofla, EPTB Vienne Conception et réalisation : Bartout P., 2014, Université d'Orléans
Figure 9 : Les dynamiques communales de densités de plans d'eau entre 1830 et 2005 dans le bassin de la Vienne The dynamics of waterbodies densities by commune between 1830 and 2005 in Vienne's basin 
la forte baisse observée depuis 1830, du plateau de Sainte-Maure à la région de Chauvigny, est le marqueur de très fortes déperditions de mares puisque ces territoires apparaissaient dépourvus d'étangs au milieu du XIX ${ }^{\mathrm{e}}$ siècle.

Cette analyse succincte montre combien les variations des densités de plans d'eau sont à elles seules insuffisantes pour définir ou non une stabilité limnique. Cette approche doit être complétée par un état des lieux qui ne cherche pas qu'à comprendre les moteurs du changement, mais également à percevoir ce qui constitue le fait ordinaire. Pour cela, nous allons étudier le type dominant de plan d'eau en 1830 et en 2005 par commune du bassin de la Vienne.

\section{L'évolution du type dominant de plan d'eau par commune}

Ces hausses ou baisses de densités ne doivent pas être interprétées comme le résultat du changement de la nature des plans d'eau, les mares devenant des étangs, par exemple, dans le cas d'une hausse de la densité. Aussi, nous nous devons de proposer une synthèse cartographique sur l'évolution du type dominant de plan d'eau entre 1830 et 2005 (figure 10).

La dominante «mare » de 1830 sur la quasi-totalité du bassin exceptée en Brenne, en Charente Limousine et sur quelques communes du HautLimousin et de Haute-Marche, a laissé place à une
Figure 10: L'évolution des dominantes de plans d'eau par commune du bassin de la Vienne entre 1830 et 2005

The evolution of waterbodies' essential features by commune in Vienne's basin between 1830 and 2005

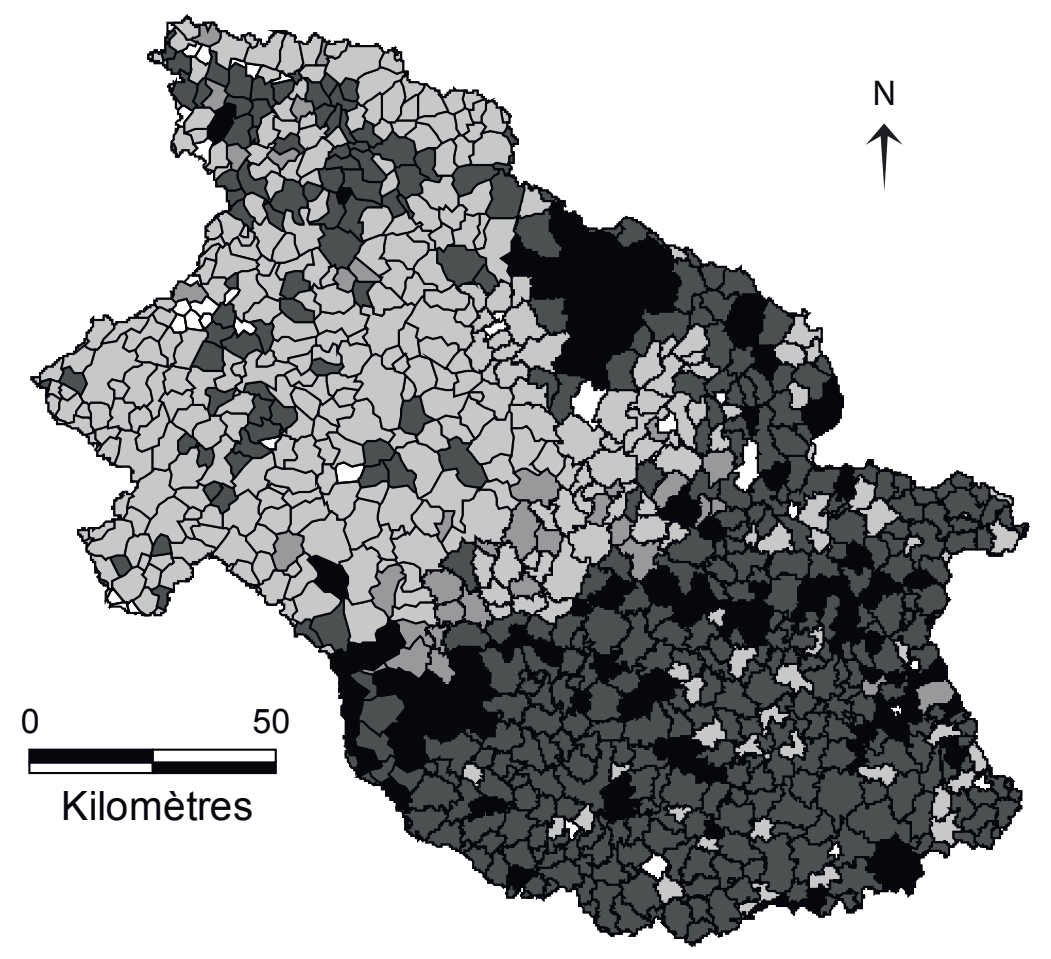

Evolution du type de plans d'eau

Dominante de mares en 1830 comme en 2005

Dominante d'étangs en 1830 comme en 2005

Passage d'une dominante d'étangs à une dominante de mares

Passage d'une dominante de mares à une dominante d'étangs

Absence de plans d'eau en 1830 ou en 2005

Données : Archives départementales, IGN BD Topo modifiée, IGN BD Geofla, EPTB Vienne Conception et réalisation : Bartout P., 2014, Université d'Orléans 
partition nette du territoire. Le sud du Limousin a massivement abandonné ses mares au profit d'étangs, alors que le nord du Poitou n'a pas connu la même évolution, même si le fait " étang » y est plus important qu'en 1830.

Afin d'affiner ces observations, nous allons mêler les différentes approches entrevues et tenter de faire apparaître des territoires de l'eau différents.

\section{LES PAYSAGES DE PLANS D'EAU DANS LE BASSIN DE LA VIENNE}

Les figures présentées ont permis de dresser un état des lieux descriptif et synthétique du fait plan d'eau dans le bassin de la Vienne. Afin de fournir un cadre de réflexion à l'aménagement et à la gestion de qualité, ces données doivent être compilées en faisant apparaître une régionalisation des plans d'eau tenant compte avant tout de la stabilité paysagère. En effet, celle-ci est le marqueur principal des reproches faits aux plans d'eau en France : les mêmes milieux présents depuis des siècles ont acquis une reconnaissance locale et ne sont pas remis en question lorsque des griefs nouveaux apparaissent. En revanche, des milieux fortement modifiés peuvent être sujets à des perceptions (réelles ou non) qui biaisent la compréhension scientifique du territoire concerné car le nouvel équilibre n’a pas encore été atteint.

Aussi, cette dernière partie distinguera dans un premier temps l'individualisation de régions limniques en fonction de leur degré de stabilité dans le bassin de la Vienne avant de décrire précisément les fonctionnements internes de chacune.

\section{L'individualisation de régions limniques}

Pour compléter les figures 9 et 10 qui constituent une synthèse pour chaque thématique étudiée, nous avons souhaité abonder la réflexion sur les plans d'eau à l'échelle nationale en tentant de différencier les territoires de l'eau, quelles que soient leurs compositions de plans d'eau et leurs densités, en fonction de leur pérennité relative (figure 11).

La présence d'une stabilité sur le long terme correspond à une logique économique et sociétale où la présence/absence du plan d'eau au sein des milieux n'est pas remise en cause. À l'inverse, les secteurs jugés peu stables révèlent des changements de logiques humaines qui impactent nécessairement le milieu. Or, ces modifications sont souvent encore trop récentes pour qu'Hommes et milieux aient pu trouver un équilibre et les tensions avec l'administration y sont plus vives qu'ailleurs.

Cette synthèse cartographique identifiant la stabilité ou l'instabilité limnique des communes situées à l'intérieur du bassin de la Vienne distingue quatre espaces géographiques aux fonctionnements différenciés, présentant d'étranges similitudes avec le découpage géologique (figure 3). L'une des logiques de la pérennité des plans d'eau au sein d'un espace serait donc en lien avec les qualités agronomiques du sous-sol et de sa perméabilité, ce que nous avons montré dans le sud du Berry (Bartout, 2014c).

\section{Les comportements lentiques des régions limniques}

Pour chaque couronne identifiée, nous présenterons donc rapidement la nature géologique des territoires concernés, et, par commodité, nous traiterons ces régions en remontant le cours de la Vienne, soit de la confluence avec la Loire jusqu'à la Montagne limousine.

\section{Les basses vallées de la Vienne et de la Creuse}

Cet espace s'étend au sein des parties aval de la Creuse et de la Vienne, jusqu'à leurs confluences respectives avec la Gartempe et le Clain. Il comprend les régions du Chinonais, du Richelais, du Mirebalais et du Châtelleraudais positionnées sur des craies, calcaires, marnes, tuffeaux et sables du Crétacé et le plateau de Sainte-Maure en Touraine, qui est surmonté d'une couche d'argiles à silex. Le point commun de cet ensemble est de n'avoir pas connu d'évolution particulière de son patrimoine limnique.

Ainsi, la partie crayeuse de cet ensemble a connu une très forte stabilité limnique sur les interfluves avec quelques mares présentes, alors que les talwegs ont subi des variations de densité plus marquées en lien avec l'urbanisation de cet espace. Le plateau argileux de Sainte-Maure, s'il apparaît aussi très stable, est marqué par une présence plus importante de petites masses d'eau, concentration permise par l’imperméabilité des argiles de surface. 


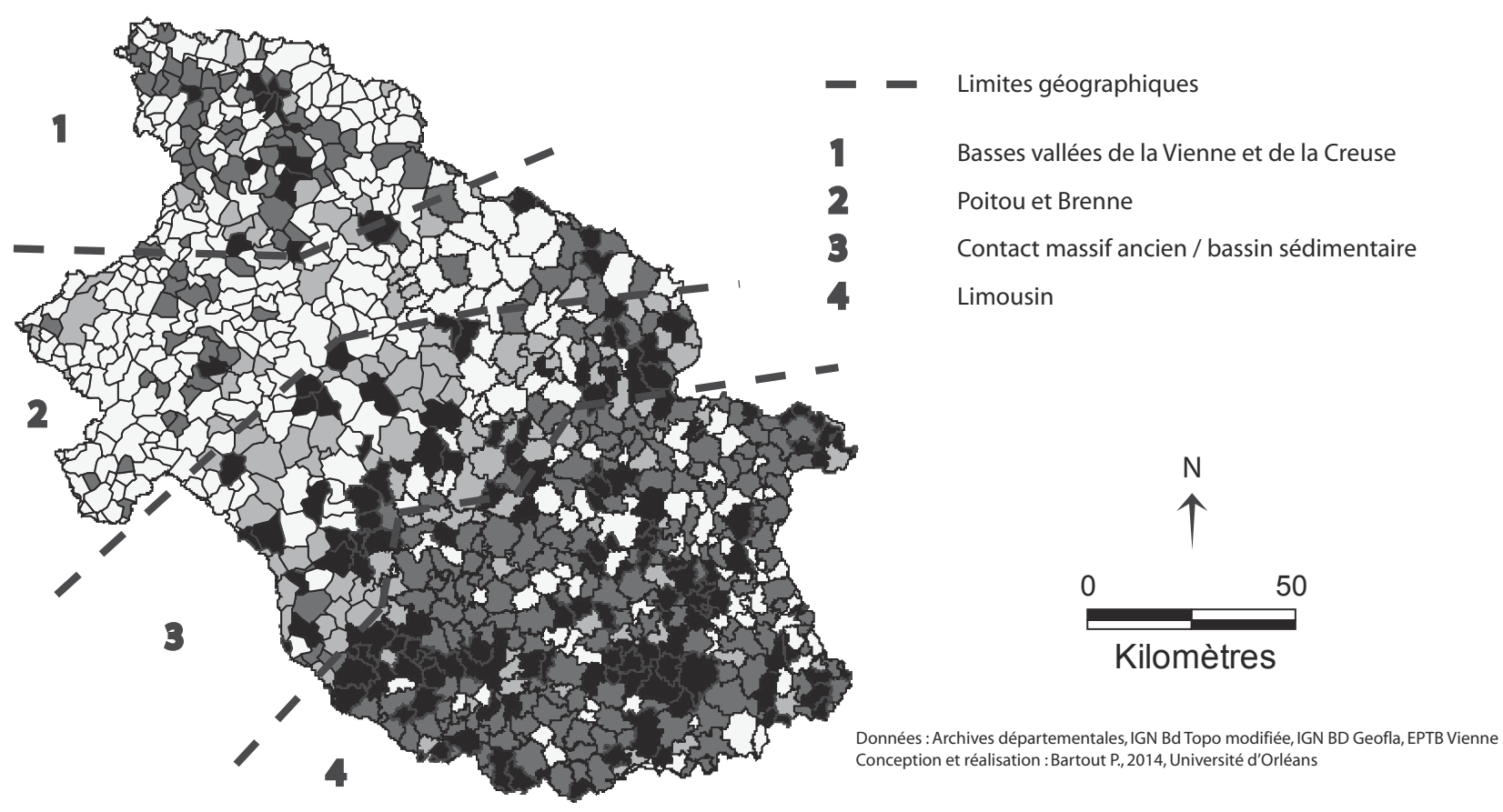

Stabilité limnique (type majoritaire et densité) par commune entre 1830 et 2005

Très forte stabilité

Stabilité de la densité mais instabilité du type de plan d'eau majoritaire

Stabilité du type de plan d'eau majoritaire mais instabilité de la densité

Double instabilité : densité et type de plan d'eau majoritaire

Figure 11 : La stabilité limnique du bassin de la Vienne Limnic stability in Vienne's basin

\section{Le Poitou et la Brenne}

Le second espace identifié correspond aux vallées poitevines du Clain (excepté sa partie amont) et de la Vienne autour de Chauvigny, auxquelles s'adjoint la transition vers le Berry qu'est la Brenne. Le point commun de cet ensemble est une extrême stabilité, tant au point de vue de la composition lentique que des densités. Des quatre ensembles, il est celui où la thématique conflictuelle liée aux plans d'eau est la plus faible.

Cette relative homogénéité ne doit pas cacher des emprises spatiales fort différentes en lien avec la nature du sous-sol et de la valorisation agricole (l'impact urbain sur l'édification ou la destruction de digues est ici peu marqué malgré la présence de la métropole poitevine). On distingue ainsi cinq sousensembles.
D'ouest en est, le premier est constitué des premiers contreforts du Massif armoricain en amont de Vasles. Il s'agit d'un isolat à fortes densités d'étangs dans une masse de mares. La présence d'étangs s'explique ici par la nature étanche du sous-sol (des leucogranites) et la faiblesse des nappes nécessitant la création de retenues d'une certaine superficie.

Le second correspond aux grandes étendues calcaires et marneuses du jurassique si peu propices à l'implantation de plans d'eau, que l'on retrouve en Champagne Berrichonne. La présence de mares est néanmoins attestée grâce au colmatage de dolines par des argiles de décalcification, modelés nommés «marchais ». Cependant, devant la mécanisation agricole des plaines poitevines, ces points bas ont été progressivement comblés, entrainant une régression des mares. 
Les trois derniers espaces font tous partie de la Brenne. Il est cependant nécessaire de distinguer la Grande Brenne, de la Petite Brenne et de la queue de Brenne (qui se poursuit au-delà du bassin de la Vienne, dans ceux de l'Indre et même du Cher).

La Grande Brenne, autour des communes de Mézières-en-Brenne et Saint-Michel-en-Brenne est la patrie des étangs par excellence du fait de son substrat imperméable et de sa platitude. Les difficultés d'écoulement des eaux ont conduit les hommes à créer une multitude de retenues, aujourd'hui valorisées par la pêche et la présence de biotopes uniques favorisant une diversité faunistique et floristique.

La Petite Brenne est séparée de sa grande sœur par la vallée de la Creuse. Si elle porte le même patronyme, le paysage y est fort différent. En effet, la place des étangs y est moins importante, la couverture forestière plus uniforme et l'élevage plus diversifié, car la pente plus prononcée permet un écoulement des eaux ne nécessitant pas l'obligation de stocker les eaux pour cultiver les terres.

Enfin, la queue de Brenne, constituée de formations superficielles détritiques moins épaisses qu'en Grande Brenne, a connu une mutation plus importante du fait lentique que ses congénères. La densité y a légèrement baissé du fait de l'abandon des mares forestières et la place des étangs s'est étendue, mais dans un but tout autre, à savoir les loisirs.

Cet agrément individuel est le moteur d'une évolution paysagère dans les deux derniers espaces traités. En fonction des prix du foncier et du degré d'accessibilité en temps depuis les principaux centres de population, la présence d'étangs va s'accentuer, accompagnée d'une destruction en masse des mares. Ce fait a pu être mis en évidence grâce à l'IRA (Indice de Renouvellement Annuel) (Bartout, 2010) consistant à comptabiliser créations et destructions de plans d'eau pour chaque territoire identifié et à diviser ce chiffre par la durée durant laquelle se sont produits ces changements. Plus l'indice est élevé et plus les modifications paysagères ont été profondes. Les territoires concernés sont donc fortement instables limniquement.

\section{Le contact massif ancien/bassin sédimentaire}

Sur ce contact entre bassin sédimentaire et massif ancien, l'encaissement des cours d'eau est un élément prépondérant car ils participent au déblayage des formations superficielles qui constituent les zones préférentielles d'implantations de plans d'eau. Cinq sous-ensembles peuvent être identifiés.

À l'ouest, la haute vallée du Clain a connu un renforcement de la prédominance des étangs au cours des dernières décennies. Ce phénomène se retrouve en Charente limousine où les mares liées au mode de mise en valeur traditionnel de l'espace, à savoir la polyculture - élevage, ont fortement diminué car l'économie s'est tournée davantage vers l'élevage bovin. Compte tenu des besoins en eau du cheptel, les hommes ont comblé les mares et mis à leur place des citernes et des étangs car ceuxci permettent une quantité et une qualité de l'eau plus importantes. Ce phénomène ne se retrouve pas plus à l'Est en Basse-Marche où la valorisation ovine des espaces agricoles a conduit à entretenir un maillage dense de mares au nord, et la grande propriété foncière au sud s'est tournée davantage vers la céréaliculture, offrant çà et là la présence d'étangs plus étendus. Cette relative grande taille de l'étang se retrouve dans le Montmorillonnais qui a connu une colonisation moyenne d'étangs contemporains venus remplacer les mares désuètes. Enfin, la région comprise entre Argenton-sur-Creuse et Aigurande a connu une double instabilité liée à la création d'étangs en très grand nombre.

\section{Le Limousin}

Cette construction effrénée d'étangs est la marque de fabrique du dernier espace identifié, à savoir le Limousin. La stabilité limnique y est quasi inexistante, notamment dans la partie nord-ouest, le Haut-Limousin. Ce territoire n'apparaît pourtant pas uni graphiquement. En effet, les marges nord (monts de Blond et d'Ambazac) et sud (monts de Châlus) ont connu avant tout une modification du type de plans d'eau dominant (de la mare à l'étang) car les densités y étaient déjà importantes en 1830. À l'inverse, la partie centrale, autour de la vallée de la Vienne en aval de Limoges et celle de la Gorre, a vu un chamboulement complet de son patrimoine lentique, passant de très faibles densités de mares à de très fortes densités d'étangs. Cette modification en profondeur se retrouve quelque peu en Haute-Marche, notamment dans la partie amont de la Petite Creuse, mais cet espace creusois est plus hétérogène dans son évolution. Enfin, il faut insister 
sur les hautes terres de la Montagne limousine où de manière générale, les densités de plans d'eau ont chuté du fait de l'abandon des mares (pêcheries), même si de nombreux lacs de barrage y ont vu le jour au cours du $\mathrm{Xx}^{\mathrm{e}}$ siècle. Néanmoins, nous décelons deux logiques au sein de ce territoire : l'une sur les parties amont planes à alvéoles où les mares sont encore présentes; l'autre au niveau des ruptures de pentes comme pour la Maulde ou le Taurion, où l'on a assisté à la destruction de pêcheries et parfois à la création d'étangs d'agrément.

\section{Conclusion}

Cette étude diachronique sur la stabilité limnique des territoires a permis de révéler l'évolution temporelle des masses d'eau présentes au sein du bassin de la Vienne. Construites, réduites, détruites, reconstruites, ces masses d'eau symbolisent une relation Hommes - milieu complexe et en constante évolution. Or, la recherche du meilleur compromis entre l'équilibre économique et l'équilibre écologique requiert un temps que la récente poussée du nombre de retenues (dans l'Ouest de la France notamment) ne permet pas encore (excepté en Poitou dans le cas de cette étude). Devant le constat d'une multiplication des espaces lentiques, il est avant tout nécessaire de limiter les nouvelles constructions et de prendre le temps de mieux comprendre les plans d'eau, afin d'identifier le ou les facteurs potentiellement perturbateurs des milieux, en toute impartialité, et de laisser les milieux et les écosystèmes s'adapter à la perturbation.

La compréhension et l'appréhension des plans d'eau sont donc forts complexes et ils ne se limitent pas à un accident de cours d'eau à l'image négative tel qu'envisagé par la réglementation en place. Les comprendre, c'est comprendre les hommes et les femmes qui les possèdent, qui les font vivre, c'est comprendre une composante ancestrale des paysages du bassin de la Vienne. Au contraire, leur effacement, tel qu'il est envisagé actuellement pour les étangs, ne constitue en rien un préalable à une bonne gestion des cours d'eau, puisqu'on feint d'ignorer, dans les bassins situés à l'amont, l'origine domestique, agricole ou industrielle des polluants relâchés par les étangs lors des vidanges. De même, la multiplication de chaînes d'étangs limitant de fait la continuité écologique entre l'amont et l'aval du cours d'eau ne doit pas faire oublier deux faits : $80 \%$ des plans d'eau se situent avant l'écoulement permanent du cours d'eau ou sur source (Bartout, 2010), et l'étang situé en tête de bassin participe très souvent à un allongement de la durée annuelle de l'écoulement dans les régions au substratum imperméable, ce qui ne saurait être le cas sans la constitution de réserves d'eau, bases mêmes des premières créations stagnustres.

\section{Bibliographie}

Aldomany M., Touchart L., Bartout P., Nedjai R., 2013. The evaporation of ponds in the French Midwest, Lakes, Reservoirs and Ponds, Romanian Journal of Limnology, vol. 7, Bucharest, p. 75-88.

Angelier E., 2000. Écologie des eaux courantes, Paris, Lavoisier, Tec \& Doc, 200 p.

Ardillier-Carras F., 2007. L'étang et son environnement humain : une géographie du rôle social, in ToucharT L. (dir.), Géographie de l'étang : des théories globales aux pratiques locales, Paris, L'Harmattan, p. 157-177.

Azaroual A., Bartout P., Touchart L., 2012. Ponds and climate, the geographical ascendancy relationship, in PANDI G., Moldovan F. (dir.), Air and water components of the environment, Cluj-Napoca, Presa Universitara Clujeana, p. 227-235.

Balabanian O., Bouet G., 1989. L'eau et la maîtrise de l'eau en Limousin, Treignac, Les Monédières, 296 p.

BARRIÈRE B. (dir.), 1998. Moines en Limousin : l'aventure cistercienne, PULIM, Limoges, 207 p.

Bartout P., 2010. Pour un référentiel des zones humides intérieures de milieu tempéré - l'exemple des étangs en Limousin (France), Sarrebruck, Éditions Universitaires Européennes, $456 \mathrm{p}$.

Bartout P., 2011 . L'apport du cadastre napoléonien aux problématiques spatiales des retenues d'eau, Revue géographique de l'Est, 51 (3-4), Nancy, 15 p.

Bartout P., 2012. Les étangs du Limousin : des zones humides face au développement durable, Les Monédières, Brive-laGaillarde, 250 p.

Bartout P. (2015a), Un potentiel lentique méconnu : la France aux 250000 étangs, in Touchart L., Bartout P., МотchaLova O. (dir.), Mieux comprendre les étangs : expériences nationales et internationales, du Berry Limousin à l'Europe orientale, Brive-la-Gaillarde, Les Monédières, p. 27-49.

BARTout P., (2015b, à paraître), Comprendre la continuité temporelle des concentrations d'étangs en France depuis la fin du $18^{\mathrm{e}}$ siècle - statistiques, cartographies, Revue Géographique de l'Est, Nancy.

Bartout P. (2015c). Les étangs du Boischaut sud : berrichons ou limousins ? Étude de cas du bassin de la Vauvre (Indre), in Touchart L., Bartout P., Motchalova O. (dir.), Mieux comprendre les étangs : expériences nationales et internatio- 
nales, du Berry Limousin à l'Europe orientale, Brive-la-Gaillarde, Les Monédières, p. 111-139.

Bartout P., Touchart L., 2013. L'inventaire des plans d'eau français : outil d'une meilleure gestion des eaux de surface, Annales de Géographie, nº 691, Paris, p. 266-289.

Bédoucha G., 2011. Les liens de l'eau : en Brenne, une société autour des étangs, Paris/Versailles, Maison des Sciences de l'homme et Quæ, 688 p.

Benarrous R., 2009. La grande Brenne aux périodes préindustrielles: contribution à l'histoire des paysages, des étangs et des relations sociétés/milieux dans une zone continentale: approches historique, archéologique et paléo environnementale, Thèse de doctorat, Université Panthéon Sorbonne de Paris, $605 \mathrm{p}$.

Bernard C., 2008. L'étang, l'homme et l'oiseau: Incidences des modes de gestion des étangs piscicoles sur les ceintures de végétation et l'avifaune nicheuse en Sologne, Brenne, Bresse, Territoire de Belfort et Champagne humide, Thèse de doctorat en géographie, ENS Lyon, 630 p.

Brunaud D., 2007. Etangs pelliculaires et réserves naturelles: principes de fonctionnement et modalités de classement, thèse de doctorat en géographie, Université de Limoges, 582 p.

Busnel L., 1985. Étangs anciens et plans d'eau contemporains en Indre-et-Loire, thèse de $3^{\text {e }}$ cycle de géographie, Université de Tours, $389 \mathrm{p}$.

Carlini M., 2006. Morphologie et hydrodynamique des plans d'eau : le cas des étangs-lacs en Limousin, Thèse de doctorat en géographie, Université de Limoges, 357 p.

Cassan M., 2006. Étangs et Révolution : l'application du décret d'assèchement des étangs du 14 frimaire an II (4 décembre 1793) dans le Limousin et la Marche, in GHZH, La production des étangs du Moyen-âge à l'époque contemporaine, Actes de la journée d'étude 2005, p. 85-102.

Cizel O., 2013. Cadre juridique, in Oertli B., Frossard P.-A. (dir.), Mares et étangs : écologie, gestion, aménagement et valorisation, Lausanne, Presses Polytechniques et universitaires romandes, p. 392-417.

Dufour S., Piégay H., 2009. From the myth of a lost paradise to targeted river restoration: forget natural references and focus on human benefits, River Research and Applications, 25, p. 568-581.

Finger-Stich A., 2013. Etangs en société : sujets versatiles, in Oertli B., Frossard P.-A. (dir.), Mares et étangs : écolo- gie, gestion, aménagement et valorisation, Lausanne, Presses Polytechniques et universitaires romandes, 13-20.

Franchomme M., 2008. Du cadastre napoléonien à la trame verte, le devenir des petites zones humides périurbaines en région Nord-Pas de Calais. Thèse de doctorat en géographie, Université de Lille, 406 p.

La Véronne (DE) C., 1967. La Brenne: histoire et traditions, Tours, Gibert-Clarey, 116 p.

Lespez L. (dir.), 2012. Paysages et gestion de l'eau. Sept millénaires d'histoire de vallées et de plaines littorales en BasseNormandie. Bibliothèque du pôle rural $n^{\circ} 3$, PU Caen MRSH, Caen, 336 p.

Maleval V., 2002. Le lac de Saint-Pardoux et l'évolution morphologique des lacs de barrage artificiel, thèse de doctorat de géographie, Université de Limoges, 357 p.

Papon P., 2007. Les plans d'eau superficiels : définitions, fonctionnement, aménagements : étude du lac Balaton, du lac de Grand-Lieu et de l'étang de Cieux, thèse de doctorat en géographie, Université de Limoges, 300 p.

Perpillou A., 1940. Le Limousin, étude de géographie physique régionale, Chartres, Durand, $257 \mathrm{p}$.

Rougier de la Bergerie J.-B. (baron), 1819. Manuel des étangs ou traité de l'art d'en construire avec économie et solidité, Paris, Audot libraire, $198 \mathrm{p}$.

Scher O., 2008. "The french pondscape: state of the art », $3^{\text {nd }}$ European pond conservation workshop, Valencia, Spain, $14-16^{\text {th }}$ may 2008.

Touchart L., 2002. Limnologie physique et dynamique, une géographie des lacs et des étangs, Paris, L'Harmattan, 395 p.

Touchart L., 2007. La définition de l'étang en géographie limnologique, in Géographie de l'étang : des théories globales aux pratiques locales, Paris, L'Harmattan, 13-53.

Touchart L., Graffouillère M. (dir.), 2004. Les étangs limousins en questions, L'AIGLE, Limoges, 179 p.

Touchart L., Bartout P., Nedjai R., 2014. La géographie limnologique en France : conjugaison de l'espace et du temps pour la compréhension des relations Homme - Milieu, Bulletin de la Société Géographique de Liège, 62, Liège, p. 81-91.

Vacher A., 1908. Le Berry, contribution à l'étude géographique d'une région française, Paris, Armand Colin, 548 p. 